\title{
Evaluation of Geriatric Patients Undergoing Hip Surgery: A Retrospective Study
}

\author{
Tuba Çatak ${ }^{1}$, Ömer Lütfi Erhan², Ayşe Belin Özer ${ }^{3}$, Mikail Kılınç ${ }^{4}$ \\ ${ }^{1}$ Department of Anesthesiology and Reanimation, Ordu University School of Medicine, Ordu, Turkey \\ ${ }^{2}$ Firat University, School of Medicine, Department of Anesthesiology and Reanimation, Elazıg, Turkey \\ ${ }^{3}$ Inonu University, School of Medicine, Department of Anesthesiology and Reanimation, Malatya, Turkey

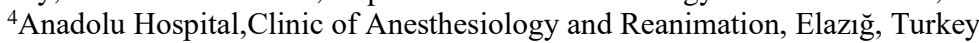

Received: 19 June 2020, Accepted: 21 October 2020, Published online: 31 December 2020

(C) Ordu University Institute of Health Sciences, Turkey, 2020

\begin{abstract}
Objective: Global improvement in the quality of life has led to a rapid expansion of the elderly population. A majority of patients undergoing hip or lower extremity surgery belong to the geriatric age group; in these patients, regional anaesthesia is generally preferred over general anaesthesia due to the common occurrence of concomitant conditions. In this study, we investigated the effect of the anaesthetic technique on mortality, morbidity, and clinical outcomes in geriatric patients undergoing hip surgery.

Methods: This study evaluated 700 patients over 65 years of age with an ASA status of III/IV who had undergone hip surgery between 2009 and 2013 at Firat University Hospital. Based on a review of patient records, 114 patients were eligible for the study. The two groups were comparable in terms of age, ASA status, gender, anaesthesia duration, haemoglobin levels at baseline and discharge, complication rates, need for post-operative intensive care unit admission, concomitant conditions, need for volume replacement, mortality rate and need for blood and blood products.

Results: Patients were divided into the general $(\mathrm{n}=76)$ and regional $(\mathrm{n}=38)$ anaesthesia groups. Pre- and post-operative haemoglobin levels were not significantly different between the two groups( $p>0.068)$ Surgery duration $(113.68 \pm 34.73 \mathrm{~min})$ and hospital stay length $(11.42 \pm 4.03$ days $)$ were statistically shorter in the regional anaesthesia group.

Conclusion: In geriatric patients undergoing hip surgery, regional anaesthesia is superior to general anaesthesia, as it results in reduced surgery duration, hospital stay length and need for blood transfusions.

Key words: Geriatrics, general anesthesia, regional anesthesia, arthroplasty, mortality rate
\end{abstract}

Suggested Citation: Catak T, Erhan OL, Ozer AB, Kilinc M. Evaluation of Geriatric Patients Undergoing Hip Surgery: A Retrospective Study. Middle Black Sea Journal of Health Science, 2020; 6(3):397-403.

Address for correspondence/reprints:

Tuba Çatak

Telephone number: $+90(541) 7464477$

ORCID-ID 0000-0001-8370-0739

E-mail: dr.tubaa@hotmail.com

DOI: $10.19127 / \mathrm{mbsjohs} .754984$

\section{Introduction}

Global improvement in the quality of life has led to a rapid expansion of the elderly population. Individuals over 65 years of age are considered elderly and those over 80 years of age are considered advanced elderly. General improvements in the quality of health care and advances in anaesthetic and surgical techniques have led to an increase in the number of the elderly undergoing anaesthesia (Turkmen and Turgut,2007, Ogurlu et al,2007; Bettelli, 2010; Deiner and Silverstein, 2011).

More than half of geriatric patients have one or more concomitant conditions, such as chronic 
obstructive pulmonary disease, diabetes mellitus, cardiac failure, or renal failure, which are associated with an increased risk of perioperative and postoperative complications (Chung et al, 1999, Aldwinckle and Montgomery, 2004, ).

The majority of patients undergoing lower extremity surgery belong to older age groups. In this population, regional anaesthetic techniques is generally preferred to general anaesthesia to avoid complications due to co-existing conditions (Celik et al, 2010). Despite the absence of clear scientific evidence on the superiority of one technique over the other, discussions regarding the advantages of regional anaesthesia have been ongoing for many years (Takmaz, 2012; Seyedi et al, 2015).

This study aimed to investigate the effect of the anaesthetic technique on morbidity and clinical outcomes in geriatric patients undergoing hip surgery. In light of recent data, a discussion regarding the efficacy and advantages of both anaesthetic methods is provided.

\section{Methods}

The study protocol was approved by the Ethics Committee for Clinical Research, Firat University Medical Faculty (Date:01.11.2012/ Decision number:18-02). This article is derived from the Republic of Turkey, our thesis registered with expertise in the national thesis center number 388667.

Data used for retrospective analyses were retrieved from case files of patients who underwent hip surgery at Firat University Hospital. We evaluated 700 patients over 65 years of age with an ASA status of III/IV and who underwent hip surgery between 2009 and 2013 at Firat University Hospital. Of these, 114 patients were eligible for the study based on adequate data in patient files (i.e. age, sex, need for volume replacement, blood transfusion, post-operative intensive care, hospital stay length, complications, haemodynamic alterations, surgery type, concomitant conditions, ASA status and duration of anaesthesia and surgery). A total of 586 patients were excluded from the analyses due to reasons including coding errors or age below 65 years.
This study included 76 patients treated with general anaesthesia and 38 with regional anaesthesia. The two groups were compared in terms of age, sex, need for volume replacement, blood transfusion, post-operative intensive care, hospital stay length, complications, haemodynamic alterations, surgery type, concomitant conditions, ASA status and duration of anaesthesia and surgery.

\section{Statistical analysis}

Statistical analyses were performed using the SPSS 15.0 software package (The Statistical Package for the Social Sciences, Chicago, IL, USA; Licence number: Z-125-3301-14). Data were recorded as mean \pm SD. Parametric data were compared using the student's t test, while chi-squared tests were used for the comparison of nonparametric data between groups. Quantitative data are expressed as mean and standard deviation minimum maximum values.A pvalue of $<0.05$ was considered significant.

\section{Results}

The two study groups, i.e., general and regional anaesthesia groups, comprised 76 and 38 patients, respectively (Figure 1). No significant age differences were noted between the two groups $(\mathrm{p}=0.075)$. The youngest and eldest patients were 65 and 104 years of age in the general anaesthesia group and 68 and 92 years of age in the regional anaesthesia groups, respectively (Table 1).

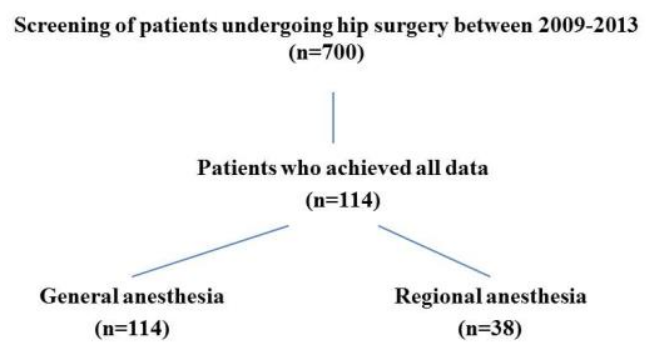

Figure 1. Patient Disposition 
Table 1.Demographic data of patients

\begin{tabular}{llcccc}
\hline Group & & Minimum & Maximum & Mean \pm SD & P value \\
\hline General & Age & 65 & 104 & $78,02 \pm 8,91$ & 0.075 \\
Anestesia & ASA classification & 3 & 4 & $3,38 \pm 0,48$ & 0.056 \\
(n=76) & Duration of anesthesia & 75 & 240 & $147,53 \pm 34,97$ & 0.066 \\
& Baseline hemoglobin (gr/d) & 9 & 17 & $12,42 \pm 1,82$ & 0.071 \\
& Hemoglobin at discarge & 7 & 14 & $9,71 \pm 1,40$ & 0.068 \\
& Volume (ml) & 1000 & 4000 & $2824,74 \pm 756,42$ & 0.081 \\
& *Lenght of hospital (day) & 3 & 50 & $13,72 \pm 7,43$ & $\mathbf{0 . 0 3 1}$ \\
& *Duration of surgery (min) & 60 & 210 & $127,17 \pm 35,48$ & $\mathbf{0 . 0 2 4} *$ \\
& Gender (F/M:48/28) & & & & \\
Regional & Age & 68 & 92 & $80,60 \pm 6,08$ & 0.075 \\
Anesthesia & ASA classification & 3 & 4 & $3,44 \pm, 50$ & 0.056 \\
(n=38) & Duration of anesthesia & 60 & 240 & $138,82 \pm 37,99$ & 0.066 \\
& Baseline hemoglobin (gr/d) & 10 & 16 & $12,73 \pm 1,67$ & 0.071 \\
& Hemoglobin at discarge & 7 & 14 & $10,23 \pm 1,54$ & 0.068 \\
& Volume (ml) & 1000 & 4100 & $2952,63 \pm 830,08$ & 0.081 \\
& $*$ Lenght of hospital (day) & 3 & 22 & $11,42 \pm 4,031$ & $\mathbf{0 . 0 3 1}$ \\
& *Duration of surgery (min) & 50 & 210 & $113,68 \pm 34,73$ & $\mathbf{0 . 0 2 4} *$ \\
& Gender (F/M:20/18) & & & & \\
\hline
\end{tabular}

$(*): \mathrm{p}<0.05$ According to Student T test or Chi-Square test

Although pre- and post-operative haemoglobin levels were not significantly different between the two groups, post-operative haemoglobin levels were lower in both groups than pre-operative levels( $\mathrm{p}=0.068$ ). However, there was a more marked decline in haemoglobin level among patients in the general anaesthesia group than among those in the regional anaesthesia group (Figure 2).

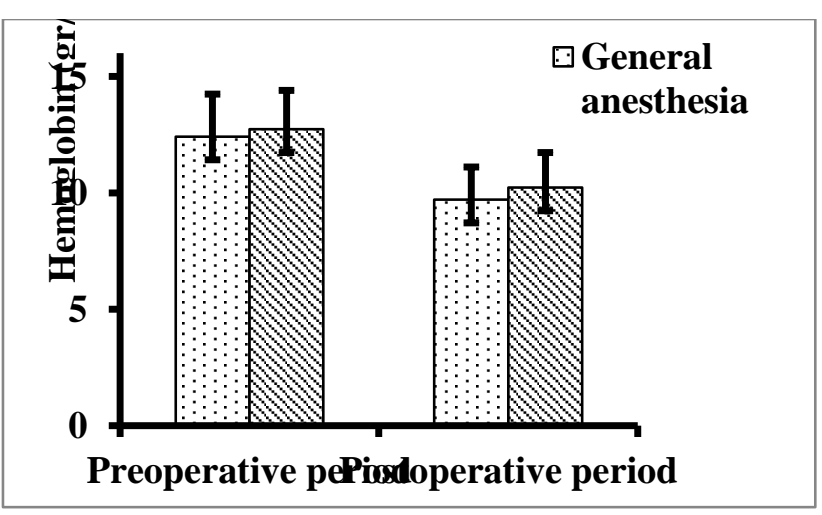

Figure 2. Comparison of pre- and post-operative haemoglobin levels in patient groups

* Right columns represent general anesthesia group, left columns represent regional anesthesia group.

The mean hospital stay lengths in the general and regional anaesthesia groups were $13.7 \pm 7.43$ and 11.4 \pm 4.03 days, respectively $(\mathrm{p}=0.031)$ (Figure 3$)$.

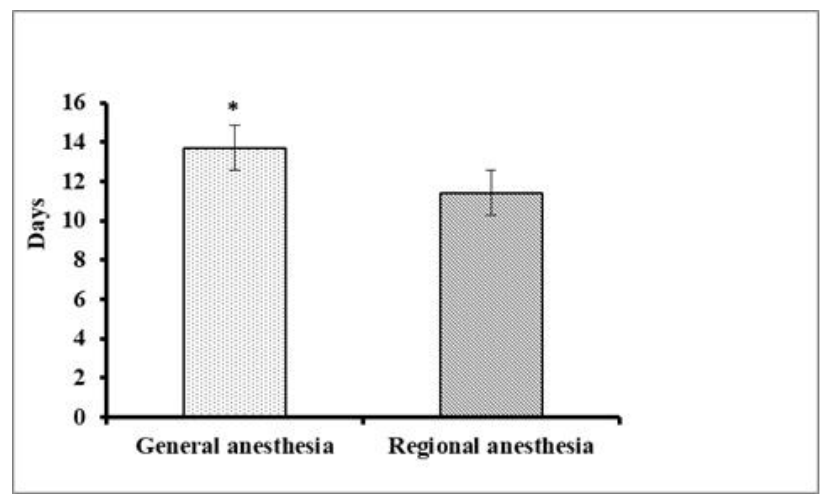

Figure 3: Comparison of hospital stay length of groups

* Right columns represent general anesthesia group, left column represent regional anesthesia group.

The mean surgery durations were $127 \pm 35.47$ and $113 \pm 34.73 \mathrm{~min}$ and the mean anaesthesia duration were $147 \pm 34.97$ and $138 \pm 37.99 \mathrm{~min}$ in the general and regional anaesthesia groups, respectively (Figure 4). $(\mathrm{p}=0.024, \mathrm{p}=0.066$ respectively $)$ 


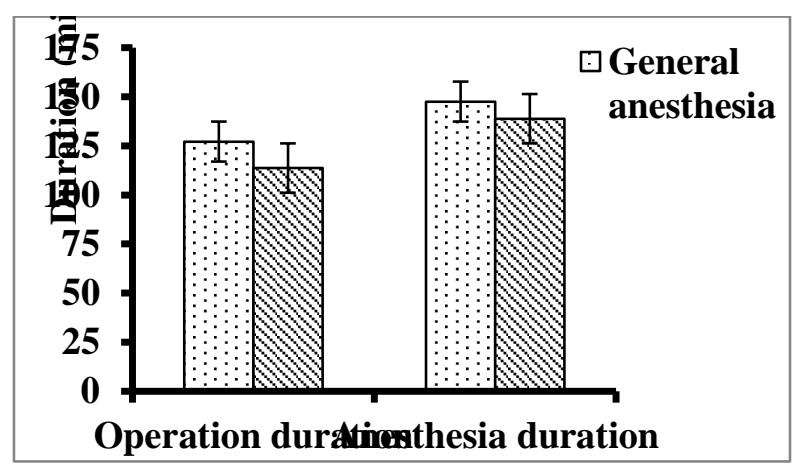

Figure 4. Comparison of duration of surgery and anaesthesia of groups

* Right columns represent general anesthesia group, left columns represent regional anesthesia group

\section{Discussion}

In our study, we found that the length of hospital stay was shortened in the regional anesthesia group between the regional anesthesia group and the general anesthesia group. Similarly, we found that the duration of surgery was shortened in the regional anesthesia group. This is evidence that regional anesthesia is more advantageous than general anesthesia. Early discharge will also protect patients from thromboembolic events. Early discharge, returning to early daily life activities will also contribute to the national economy.

For study purposes, participants were classified in two groups: those undergoing regional anaesthesia and general anaesthesia. The two groups were examined and compared in terms of age, sex, need for volume replacement, blood transfusion, postoperative intensive care, hospital stay length, complications, haemodynamic alterations, surgery type, concomitant conditions, ASA risk score and duration of anaesthesia and surgery.

In the study by Polanczyk et al. (2001) which involved 4315 patients over 50 years of age undergoing elective non-cardiac surgical procedures between 1989 and 1994, the age-adjusted mortality and morbidity rates were reported as follows: mortality rate in individuals between 50-59, 60-69, $70-79$ and $>80$ years of age were $3 \%, 5 \%, 9 \%$ and $>26 \%$, respectively. The corresponding figures for morbidity rate were $4.3 \%, 5.7 \%, 9.6 \%$ and $12.5 \%$, respectively.

In our study of 114 patients over 65 years of age, $64.91 \%$ were over 75 years of age and $21.1 \%$ were over 85 years of age. The mean age in our patient group was lower than that in several previous reports involving geriatric patients with femoral fracture.

In a retrospective cohort analysis by Franzo et al. (2005) which involved 6629 patients undergoing surgery in different centres between 1996 and 2001, the in-hospital mortality rate was $5.4 \%$, mortality rates at 6 and 12 months after discharge were $20.0 \%$ and $25.3 \%$, respectively. In addition, there was a significant decline in mortality rates between 1996 and 2001, which was confirmed in a multivariate model. The authors propose that the single most important factor accounting for this decline is the reduced workload of surgical units.

Foss and Kehlet, (2005) in their meta-analysis on mortality in patients with hip fractures undergoing surgery in different European centres, found a 30-day mortality rate of $10 \%$ and a 1 -year mortality rate of $25 \%$. The mortality rate was $7.8 \%(9 / 114)$ in that study. In our study, the in-hospital mortality rate was $7.9 \%$, consistent with that of the published data.

In a 1984 study, McKenzie et al (1984) reported a lower rate of post-operative mortality rate in a limited number of patients undergoing regional anaesthesia than in those undergoing general anaesthesia. In another study examining the association between the anaesthetic technique and mortality in elderly patients undergoing hip surgery, the mortality rate was $14.61 \%$ in general anesthesia group while it was $9.09 \%$ in regional anesthesia, and the relative and absolute risk of mortality was higher in the general anaesthesia group, whereas the causes of death (cardiovascular disease or infection) did not significantly differ (Melendez et al, 2009).

The comparison of mortality between regional and general anaesthesia group in our study showed a mortality rate of $7.9 \%$ in the regional anaesthesia group, with three cases of mortality in 38 patients. The corresponding mortality rate in the general anaesthesia group was $7.9 \%$, with six cases of mortality in 76 patients. These results suggest that the anaesthetic technique does not affect mortality rates.

Meyer et al. (2009) found a significantly increased risk of mortality in patients with hip fracture who had low mental status scores, two or more concomitant conditions and a history of being unable to walk outside of the home before the fracture. No such increase was noted in patients without these risk factors.

Svensson et al. (1996) found an association between the 1-year post-operative mortality rate and concomitant conditions before surgery. Patients with no concomitant health conditions had no mortality, whereas those with one or two concomitant conditions had a mortality rate of $14 \%$ and those with three or four conditions had a mortality rate of $24 \%$.

In this study on geriatric patients who underwent hip surgery in a 4-year period, in-hospital mortality rates were estimated. Fifteen of our 114 participants had no concomitant conditions. All nine patients who 
died had at least one concomitant condition, eight had more than two conditions and one had only a single concomitant condition.

Previous studies suggest that epidural anaesthesia in patients undergoing total hip replacement could reduce blood loss and prevent intraoperative hypertension. It may also reduce the incidence of reoperation in those undergoing vascular surgery by providing adequate tissue perfusion. Such reported advantages support the utilisation of regional anaesthesia and analgesia in the elderly (Jin and Chung, 2001). Regional anaesthesia is considered to confer the lowest risk of post-operative hypoxaemia, consequently reducing pulmonary oedema and blood loss and allowing early diagnosis of mental status changes. Furthermore, the effect of spinal anaesthesia on lowering central venous pressure should be emphasised (Rodgers et al, 2000).

In this study, rates of post-operative reduction in haemoglobin levels were similar between the regional and general anaesthesia groups. The proportions of patients experiencing bleeding were also comparable. However, the reduction in haemoglobin levels was less marked in the regional anaesthesia group.

Maurer et al. (2007) reported significantly reduced surgical time, intra- and post-operative blood loss and need for transfusions in patients undergoing spinal anaesthesia, suggesting that spinal anaesthesia may be superior to general anaesthesia for patients undergoing unilateral hip surgery.

Neuman et al. (2014) divided patients undergoing hip surgery based on the type of anaesthetic technique used, i.e. regional and general anaesthesia groups. The two groups were compared with regard to 30-day mortality and hospital stay length. There was no significant difference in the former, whereas the latter was shorter among regional anaesthesia patients.

In a review by Liu Y. et al. (2017) They emphasized that anesthesia management in the elderly started with preoperative examination and multidispliner should be approached while making preoperative preparation. Perioperatively, they emphasized that it should be taken to surgery with equipment intended for multiorgan protection. For example, they emphasized that it would be better to plan the operation with advanced monitoring techniques.

Should we avoid general anesthesia in elderly patients? entitled of Strom et al. (2014) They stated that postoperative cognitive dysfunction is more common in regional anesthesia in general anesthesia. They emphasized that minimal sedation and regional anesthesia should be preferred in elderly patients whenever possible. If there is no contraindication in our clinic, the first choice is regional anesthesia in the elderly.

In a study by Patel et al., (2018) They compared general anesthesia and regional anesthesia techniques in elderly patients. They stated that they did not see any difference in terms of mortality and morbidity in patients receiving general anesthesia.

In a study by Chen et al., (2019) They compared different anesthesia techniques in terms of mortality and morbidity in hip fracture in elderly patients. In terms of 30-day mortality, they did not see any difference between the group receiving general and regional anesthesia. However, they observed that postoperative complications were higher in the group receiving general anesthesia.They observed that the frequency of complications such as postoperative respiratory failure, longer hospital stay, longer stay in intensive care unit was higher in the general anesthesia group. In our study, similarly, we detected fewer complications in patients undergoing regional anesthesia.

Similar to previous reports, patients in the regional anaesthesia group in our study required fewer blood transfusions and spent less time in the hospital compared with those in the general anaesthesia group.

The main results of our study include the following:

No difference between patients undergoing hip surgery with general or regional anaesthesia in terms of age, sex, ASA risk score, anaesthesia duration, volume replacement, complications and need for intensive care. The surgery duration was shorter among regional anaesthesia patients than among general anaesthesia patients. Despite the absence of a significant difference between pre- and postoperative haemoglobin values, the reduction was less marked in regional anaesthesia patients.The frequency of concomitant conditions was comparable across the study groups.Blood transfusion requirements were similar between the two groups, although patients in the regional anaesthesia group required less blood transfusion. The two groups were comparable in their need for intensive care.Patients in the regional anaesthesia group had a shorter hospital stay length.

\section{Conclusion}

No difference between patients undergoing hip surgery with general or regional anaesthesia in terms of age, sex, ASA risk score, anaesthesia duration, volume replacement, complications and need for intensive care. The surgery duration was shorter among regional anaesthesia patients than among generalanaesthesia patients. Despite the absence of a 
significant difference between pre- and postoperative haemoglobin values, the reduction was less marked in regional anaesthesia patients.The frequency of concomitant conditions was comparable across the study groups.Blood transfusion requirements were similar between the two groups, although patients in the regional anaesthesia group required less blood transfusion. The two groups were comparable in their need forintensive care.Patients in the regional anaesthesia group had a shorter hospital stay length

In the ligt of all these results, regional anaesthesia seems to offer certain advantages over general anaesthesia in elderly patients undergoing hip surgery with respect to hospital stay length, surgery duration and the need for blood transfusions.

Ethics Committee Approval: Ethics Committee for Clinical Research, Firat University Medical Faculty (Date:01.11.2012/ Decision number:18-02)

Peer-review: Externally peer-reviewed.

Author Contributions: Concept- T.C, A.B.O, M.K; Design- A.K., T.C, O.L.E, A.B.O, M.K.; MaterialsT.C., O.L.E.; Data Collection and Processing- T.C, O.L.E., A.B.O., M.K.; Literature Review- T.C., O.L.E., A.B.O.; Writing- T.C, O.L.E., A.B.O.; Critical Review- T.C., A.B.O.

Conflict of Interest: No conflict of interest was declared by the author.

Financial Disclosure: The author declared that this study hasn't received no financial support.

\section{References}

Aldwinckle RJ, Montgomery JE. Unplanned admission rates and postdischarge complications in patients over the age of 70 following day case surgery. Anaesthesia; 2004;59(1),57-9.

Bettelli G. Anaesthesia for the elderly outpatient: preoperative assessment and evaluation, anaesthetic technique and postoperative pain management. Curr Opin Anaesthesiol; 2010;23(6),726-31.

Chen Dong Xu, Yang Lei, Ding Lin, Li Shi Yue, Qi $\mathrm{Ya} \mathrm{Na}$, Li Qian. Perioperative outcomes in geriatric patients undergoing hip fracture surgery with different anesthesia techniques: A systematic review and meta-analysis Medicine (Baltimore); 2019 Dec; 98(49), e18220. Published online 2019 Dec 10. doi: 10.1097/MD.0000000000018220

Chung F, Mezei G, Tong D. Adverse events in ambulatory surgery. A comparison between elderly and younger patients. Can J Anaesth;1999; 46(4), 309-321.
Celik F, Tufek A, Yildirim ZB, Karaman H, Baykan H, Olmez Kavak G et al. Spinal anesthesia with small dose bupivakain in the highly elderly patient J Clin Exp Invest; 2010;1(3),214-15.

Deiner S, Silverstein JH. Anesthesia for geriatric patients. Minerva Anestesiol;2011;77(2),180-9

Franzo A, Francescutti C, Simon G. Risk factors correlated with post-operative mortality for hip fracture surgery in the elderly: a population-based approach. Eur J Epidemiol; 2005;20(12), 985-91.

Foss NB, Kehlet H. Mortality analysis in hip fracture patients: implications for design of future outcome trials. Br J Anaesth; 2005; 94(1), 24-9.

Jin F, Chung F. Minimizing perioperative adverse events in the elderly. Br J Anaesth; 2001; 87(4),608-24.

Liu Y, Xiao W, Meng LZ, Wang TL. Geriatric Anesthesia-related Morbidity and Mortality in China: Current Status and Trend. Chin Med J; 2017;130(22),2738-49.

Maurer SG, Chen AL, Hiebert R, Pereira GC, Di Cesare PE. Comparison of outcomes of using spinal versus general anesthesia in total hip arthroplasty. Am J Orthop; 2007; 36(7),E101E106.

McKenzie PJ, Wishart HY, Smith G. Long-term outcome after repair of fractured neck of femur. Comparison of subarachnoid and general anaesthesia. Br J Anaesth; 1984;56(6),581-5

Melendez HJ, Mercado A, Cobos JH. Morbimortality in major hip surgery: A study of the efficacy of selective subarachnoideal spinal anaesthesia compared to balanced general anaesthesia. A controlled clinical trial. Rev Col Anest;2009;37(3),189-201

Meyer HE, Tverdal A, Falch JA, Pedersen JI. Factors associated with mortality after hip fracture. Osteoporos Int; 2000;11,228-32 doi.org/10.1007/s001980050285

Neuman MD, Rosenbaum PR, Ludwig JM, Zubizarreta JR, Silber JH. Anesthesia technique, mortality, and length of stay after hip fracture surgery. JAMA; 2014;311(24),2508-17

Ogurlu M, Sen S, Ugur B, Discigil G, Aydın ON, Gursoy F. Evaluation of Hypotension Development Due to Spinal Anesthesia in Patients Over 65 Years of Age Turkish Journal of Geriatrics; 2007; 9(3), 126-9.

Patel V, Champaneria R, Dretzke J, Yeung J . Effect of regional versus general anaesthesia on postoperative delirium in elderly patients undergoing surgery for hip fracture: a systematic review BMJ Open;.2018; 8(12),e020757. doi: 10.1136/bmjopen-2017-020757 
Polanczyk CA, Marcantonio E, Goldman L, et al. Impact of age on perioperative complications and length of stay in patients undergoing noncardiac surgery. Ann Intern Med; 2001; 134(8), 637-43.

Rodgers A, Walker N, Schug S, et al. Reduction of postoperative mortality and morbidity with epidural or spinal anaesthesia: results from overview of randomised trials. BMJ;2000;321(7275),1493-7.

Seyedi HR, Mahdian M, Khosravi G, Bidgoli MS, Mousavi SG, Razavizadeh MR, et al. Prediction of mortality in hip fracture patients:role of routine blood tests. Archives of bone and joint surgery; 2015;3(1),51-55

Strom C,Rasmussen LS,Sieber FE Should general anaesthesia be avoided in the elderly? Anaesthesia; 2014 Jan; 69(Suppl 1), 35-44.

Svensson O, Stromberg L, Ohlen G, Lindgren U. Prediction of the outcome after hip fracture in elderly patients. J Bone Joint Surg Br; 1996; 78,115-8.

Takmaz SA. Anesthesia in Orthopedic Surgery In: Kegik H, Alkış H, Yorükoglu D, Alanoglu Z (eds). Basic Anesthesia 1st edition, Gunes Medical Bookstores; Ankara 2012, pp 653-84

Turkmen A, Turgut N. Geriatric Anesthesia. Turkish Journal of Geriatrics 2007; 10(1), 49-56. 